\title{
Convergence analysis of three parareal solvers for impulsive differential equations
}

\author{
Zhiyong Wang ${ }^{\mathrm{a}}$, Liping Zhang ${ }^{\mathrm{b}, *}$ \\ a School of Mathematical Sciences, University of Electronic Science and Technology of China, Chengdu, \\ Sichuan 610731, China \\ b School of Science, Sichuan University of Science and Engineering, Zigong, Sichuan 643000, China
}

*Corresponding author, e-mail: zlp640602@163.com

Received 27 Apr 2018

Accepted 9 Sep 2018

\begin{abstract}
We are interested in using the parareal algorithm consisting of two propagators, the fine propagator $\mathscr{F}$ and the coarse propagator $\mathscr{G}$, to solve the linear differential equations $u^{\prime}(t)+A u(t)=f$ with stable impulsive perturbations $\Delta u(t)=\alpha u\left(t^{-}\right)$for $t=\tau_{l}$, where $\alpha \in(-2,0), \Delta u(t)=u\left(t^{+}\right)-u\left(t^{-}\right)$, and $l \in \mathbb{N}$. We consider the case that $A$ is a symmetric positive definite matrix and $\mathscr{G}$ is defined by the implicit Euler method. In this case, provable results show that the algorithm possesses constant convergence factor $\rho \approx 0.3$ if $\alpha=0$ and $\mathscr{F}$ is an L-stable numerical method. However, if $\mathscr{F}$ is not L-stable, such as the widely used Trapezoidal rule, it unfortunately holds that $\rho \approx 1$ if $\lambda_{\max } \gg 1$, where $\lambda_{\max }$ is the maximal eigenvalue of $A$. We show that with stable impulses the parareal algorithm possesses constant convergence factors for both the L-stable and A-stable $\mathscr{F}$-propagators, such as the implicit Euler method, the Trapezoidal rule and the 4th-order Gauss Runge-Kutta method. Sharp dependence of the convergence factor of the resulting three parareal algorithms on the impulsive parameter $\alpha$ is derived and numerical results are provided to validate the theoretical analysis.
\end{abstract}

KEYWORDS: parallel in time, Runge-Kutta method, numerical experiments

MSC2010: 65R20 45L05 65L20

\section{INTRODUCTION}

The qualitative research of impulsive differential equations (IDEs) began in 1960 with the work of Mil'Man and Myshkis ${ }^{1}$. The wide real world applications explain the growing interest of many authors in the investigation of these equations. The theory of IDEs is emerging as an important and active area of investigation, since it is much richer than the corresponding theory of the ordinary differential equations (ODEs) without impulsive effects. Furthermore, the IDEs appear to represent a natural framework for mathematical modellings of several real world phenomena. For example, systems with impulsive effects have applications in physics, biotechnology, industrial robotics, radiotechnology, pharmacokinetics, population dynamics, ecology, optimal control, microorganism reproduction, economics, production theory, and many others. The reader interested in the background of IDEs can refer to the monographs ${ }^{2-4}$. However, the mathematical theory of systems with impulse effect has developed rather slowly, owing to the considerable difficulties of a theoretical and technical nature related to the specific character of mass systems. In addition, the presence of impulses complicates the stability analysis of the system; for example, impulses not only can make a stable system become an unstable one, but can also make an unstable one become a stable one.

Recently, there have been intensive studies on the qualitative behaviour of solutions of impulsive differential equations ${ }^{2-7}$. However, for many IDEs, even in the purely linear case, an analytic solution of simple form is often unavailable. In face of this situation, numerical computation is a natural choice $^{8-11}$. The goal of this study is to study the parareal algorithm proposed by Lions et $\mathrm{al}^{12}$ for a class of representative IDEs:

$$
\begin{aligned}
u^{\prime}(t)+A u(t) & =f(t), t \neq \tau_{l}, \\
\Delta u(t) & =\alpha u\left(t^{-}\right), t=\tau_{l},
\end{aligned}
$$

where $\Delta u(t)=u\left(t^{+}\right)-u\left(t^{-}\right), 0<\tau_{1}<\tau_{2}<\cdots$, and $A \in \mathbb{R}^{m \times m}$ is symmetric positive definite (SPD). This algorithm is used to realize parallel-in-time compu- 
tation of differential equations. The parareal algorithm is defined by two time propagators, namely, $\mathscr{G}$ and $\mathscr{F}$, which are, respectively, associated with a large step-size $\Delta T$ and a small step-size $\Delta t$, when $\Delta T=J \Delta t$ for an integer $J \geqslant 2$. These two propagators proceed in a prediction-correction manner: the $\mathscr{G}$-propagator generates rough approximations to the solution of differential equations of interest on the coarse time grids $\left\{T_{n}\right\}_{n=1}^{N}, N=T / \Delta T$, and then the $\mathscr{F}$-propagator generates accurate approximations on the fine time grids $\left\{T_{n+j / J}\right\}_{j=1}^{J}$ by using the rough approximation at $t=T_{n}$ as the initial value. Since such rough approximations are available for all the coarse time grids, the computation of the $\mathscr{F}$ propagator over all the subintervals $\left\{\left[T_{n}, T_{n+1}\right]\right\}_{n=0}^{N-1}$ can start simultaneously. The last step is to perform the so-called coarse grid correction, the heart part of the parareal algorithm, to update the rough approximations on the coarse time grids via iterations. The parareal algorithm, the closely related variants are the SDC-parareal algorithm ${ }^{13}$ and the PFASST algorithm ${ }^{14}$, attracts considerable attention in the past years. Particularly the convergence properties of this algorithm have been analysed for differential equations arising from different areas ${ }^{15-24}$. However, there are no results about this algorithm for differential equations with impulses.

Throughout this study, we consider the case that the impulse points $\left\{\tau_{l}\right\}_{l \geqslant 1}$ are equally spaced, $\tau_{l+1}-$ $\tau_{l}=\tau$ for all $l \geqslant 0$ with $\tau_{0}=0$. We analyse the convergence properties of the parareal algorithm in the case that a single large step-size $\Delta T$ contains $p$ impulses, $p \geqslant 1$ is an integer, $\Delta T=p \tau$. In this case, we apply the $\mathscr{G}$-propagator with the large step-size $\Delta T$ to the problem

$$
\begin{aligned}
u^{\prime}(t)+A u(t) & =f(t), t \neq \tilde{\tau}_{l}, \\
\Delta u(t) & =\beta u(t), t=\tilde{\tau}_{l},
\end{aligned}
$$

where $\beta=(1+\alpha)^{p}-1$ and $\tilde{\tau}_{l}=\tau_{p l}$ for $l \geqslant 1$. Clearly, this model is different from (1), but the iterates generated by the parareal algorithm still converge to the solution of (1) at the coarse time grids, which will be explained in the next section.

For the regular ODEs, $\alpha=0$ in (1), both models (1) and (2) are the same, and the convergence properties of the parareal algorithm bas been studied extensively in the past years ${ }^{15,16,19,22,24}$. The main finding is that the convergence factor $\rho(J)$ of the parareal algorithm satisfies

$$
\rho(J) \approx 0.3, \quad J \geqslant 2, \sigma(A) \subseteq[0, \infty),
$$

if we choose for $\mathscr{G}$ the implicit Euler method and for
$\mathscr{F}$ some L-stable numerical method such as the implicit Euler method, the 2nd-order singly diagonal implicit RK method, or the TR/BDF2 method (the ode23tb solver in MATLAB). This result implies that for SPD problem we can expect constant convergence factor for the parareal algorithm independent of the mesh ratio $J$ and the eigenvalue distribution of the coefficient matrix $A$. This independence is important to guarantee that refining $\Delta t$ and/or $\Delta x$ to improve the accuracy of the converged solution does not deteriorate the convergence rate. However, this result does not hold universally when $\mathscr{F}$-propagator is only A-stable, e.g., Trapezoidal rule and 4th-order Gauss RK method, which will be explained later.

Interestingly, it is found in this study that the parareal algorithm with an A-stable $\mathscr{F}$-propagator can possess constant convergence factor when stable impulses occur, $\alpha \neq 0$. We give rigorous proof for this statement for three representative parareal algorithms: parareal-Euler, parareal-TR, and parareal-Gauss4, which use for $\mathscr{G}$ the implicit Euler method and for $\mathscr{F}$ the implicit Euler method, the Trapezoidal rule, and the 4th-order Gauss RK method. The accuracy of the converged solution of these three parareal algorithms is of order 1, 2 , and 4 , respectively. We derive sharp bounds of the convergence factors of these three parareal algorithms, which only depend on the quantity $\gamma:=$ $|1+\alpha|^{-p}$. In other words, there exists a quantity $\hat{\rho}(\gamma)$ such that

$$
\rho(J) \leqslant \hat{\rho}(\gamma), \quad J \geqslant 2, \sigma(A) \subseteq[0, \infty),
$$

where $\hat{\rho}(\gamma)$ approaches 1 as $\gamma \rightarrow 1(\alpha=0$ or $\alpha=$ $-2)$ and $\hat{\rho}(\gamma)<1$ for $\gamma>1(\alpha \in(-2,0))$. Our numerical results presented in the last section show that the upper bound $\hat{\rho}(\gamma)$ is sharp and predicts the convergence rates of the three parareal algorithms very well.

\section{THE PARAREAL ALGORITHM}

In this section, we present details for the parareal algorithm with some preliminary results about the convergence property of the parareal algorithm. For problem (1), with two numerical propagators $\mathscr{F}$ and $\mathscr{G}$, we denote by $\mathscr{F}\left(T_{n}, u_{n}, \Delta t\right)$ the approximate solution of (1) at $t=T_{n}+\Delta t, \mathscr{F}\left(T_{n}, u_{n}, \Delta t\right) \approx u\left(T_{n}+\right.$ $\Delta t$ ), obtained by applying $\mathscr{F}$ with the initial value $u_{n}$ at $t=T_{n}$ and the small step-size $\Delta t$. Similarly, we denote by $\mathscr{G}\left(T_{n}, u_{n}, \Delta T\right)$ the approximate solution of (2) at $t=T_{n}+\Delta T$ obtained by applying $\mathscr{G}$ with the initial value $u_{n}$ at $t=T_{n}$ and the large step-size $\Delta T$. We divide the whole time-interval $[0, T]$ into 
$N$ large time-intervals $\left[T_{n}, T_{n+1}\right], n=0,1, \ldots, N-1$ with a uniform size, $T_{n+1}-T_{n}=\Delta T=T / N$. For each large time-interval $\left[T_{n}, T_{n+1}\right]$, we divide it into $J \geqslant 2$ small time-intervals $\left[T_{n+j \Delta T / J}, T_{n+(j+1) \Delta T / J}\right]$, $j=0,1, \ldots, J-1$. We designate by the symbols $\ominus$ and $\oplus$ the sequential and parallel parts of the parareal algorithm, respectively. Then the parareal algorithm is given as Algorithm 1 .

Algorithm 1 Parareal algorithm.

$\ominus$ Initialization: $u_{n+1}^{0}=\mathscr{G}\left(T_{n}, u_{n}^{0}, \Delta T\right)$ with $u_{0}^{0}=u_{0}$, $n=0,1, \ldots, N-1$. For $k=0,1, \ldots$ :

$\oplus$ Step 1: On each subinterval $\left[T_{n}, T_{n+1}\right]$, com-

pute $\tilde{u}_{n+(j+1) / J}=\mathscr{F}\left(T_{n+j / J}, \tilde{u}_{n+j / J}, \Delta T / J\right)$ for

(1) with initial value $\tilde{u}_{n}=u_{n}^{k}, T_{n+j / J}=T_{n}+$ $j \Delta T / J$, and $j=0,1, \ldots, J-1$;

$\ominus$ Step 2: perform sequential corrections $u_{n+1}^{k+1}=$ $\mathscr{G}\left(T_{n}, u_{n}^{k+1}, \Delta T\right)+\tilde{u}_{n+1}-\mathscr{G}\left(T_{n}, u_{n}^{k}, \Delta T\right)$, where $u_{0}^{k+1}=u_{0}, n=0,1, \ldots, N-1$, and $\mathscr{G}$ is applied to (2);

$\ominus$ Step 3: If $\left\{u_{n}^{k+1}\right\}_{n=1}^{N}$ satisfy stopping criteria, stop the iterations; otherwise, go to Step 1.

Clearly, the argument $\tilde{u}_{n+1}$ can be written as $\tilde{u}_{n+1}=$ $\mathscr{F}^{J}\left(T_{n}, u_{n}^{k}, \Delta t\right)$ and Algorithm 1 can be written compactly as

$$
\begin{aligned}
u_{n+1}^{k+1}=\mathscr{G} & \left(T_{n}, u_{n}^{k+1}, \Delta T\right) \\
& +\mathscr{F}^{J}\left(T_{n}, u_{n}^{k}, \Delta t\right)-\mathscr{G}\left(T_{n}, u_{n}^{k}, \Delta T\right),
\end{aligned}
$$

where $\Delta t=\Delta T / J$ and $\mathscr{F}^{J}\left(T_{n}, u_{n}^{k}, \Delta t\right)$ denotes a value calculated by applying successively $J$ steps of the fine propagator $\mathscr{F}$ to (1) with initial value $u_{n}^{k}$ and the fine step size $\Delta t$. Clearly, upon convergence we have $u_{n+1}^{\infty}=\mathscr{F}^{J}\left(T_{n}, u_{n}^{\infty}, \Delta t\right)$, the approximation at the coarse grids will have achieved the accuracy of the fine propagator $\mathscr{F}$ with the small step-size $\Delta t$. Hence, the $\mathscr{G}$-propagator has no effects on the accuracy of the converged solution and this explains why it makes sense to apply the $\mathscr{G}$-propagator to a completely different model, such as (2).

Theorem 1 Let $\mathscr{R}_{f}(z)$ and $\mathscr{R}_{\mathrm{g}}(z)$ be the stability functions of the two numerical propagators $\mathscr{F}$ and $\mathscr{G}$ applied to the IDEs (1) and (2), respectively. Let $\sigma(\Delta T A)=\left\{\Delta T \lambda_{1}, \ldots, \Delta T \lambda_{m}\right\}$ be the spectrum of the matrix $\triangle T A$. Then, the errors $\mathrm{e}_{n}^{k}=u_{n}^{k}-u_{n}$ of the parareal algorithm satisfy

$$
\sup _{n}\left\|V \mathrm{e}_{n}^{k}\right\|_{\infty} \leqslant \rho^{k}(J) \sup _{n}\left\|V \mathrm{e}_{n}^{0}\right\|_{\infty},
$$

provided $\left|\mathscr{R}_{\mathrm{g}}(z)\right| \leqslant 1$ for all $z \in \sigma(\Delta T A), V \in \mathbb{R}^{m \times m}$ consists of the eigenvectors of $A$, and $k \geqslant 1$ is the iteration index. The argument $\rho:=\max _{z \in \sigma(\triangle T A)} \mathscr{K}(z, J)$ is the convergence factor of the parareal algorithm, where $\mathscr{K}$ is the convergence factor corresponding to a single eigenvalue, or contraction factor, is defined by

$$
\mathscr{K}(z, J)=\frac{\left|\mathscr{R}_{\mathrm{f}}(z / J)-\mathscr{R}_{\mathrm{g}}(z)\right|}{1-\left|\mathscr{R}_{\mathrm{g}}(z)\right|} .
$$

Proof: The proof of this theorem follows directly from the analysis of Ref. 15.

Throughout this study, we choose for the $\mathscr{G}$ propagator the implicit Euler method. This is a common choice in the field of the parareal algorithm, since the propagator $\mathscr{G}$ needs a large stepsize, and therefore needs to be strongly stable. Furthermore, the propagator $\mathscr{G}$ should be cheap for implementation because it dominates the cost of the coarse grid correction for each iteration. For the $\mathscr{F}$-propagator, we consider three RK methods; the implicit Euler method, the Trapezoidal rule, and the 4th-order Gauss method. The resulting three parareal algorithms are denoted by parareal-Euler, parareal-TR, and parareal-Gauss4, respecively. To derive the stability function of the three RK methods applied to the impulsive differential equations, we consider the scalar model problem

$$
\begin{aligned}
u^{\prime}(t)+\lambda u(t) & =0, \quad t \in\left(\tau_{l}, \tau_{l+1}\right), \\
\Delta u(t) & =\alpha u\left(t^{-}\right), \quad t=\tau_{l},
\end{aligned}
$$

where $\tau_{1}=\tau$ and $\tau_{l+1}-\tau_{l}=\tau$ for $l \geqslant 1$. We assume that $\tau=q \Delta t$, the impulse distance $\tau$ contains $q$ small step-sizes, and $\Delta T=p \tau$. Thus $J=p q$. We illustrate the relation between the numerical solutions computed by using the small step-size $\Delta t$ at two adjacent impulses, $t=\tau_{l-1}$ and $\tau_{l}$. Denote by $u_{l, j / q}$ and $u_{l, 0}$ the approximations of $u\left(T_{l+j / q}\right)$ and $u\left(T_{l}+0\right)$, where $T_{l+j / q}=T_{l}+j \Delta t$ and $T_{l}=l \tau$ with $0 \leqslant j \leqslant q, l=0,1, \ldots, N_{\tau}=T / \tau$. Thus for the implicit Euler method we have

$$
\begin{aligned}
u_{l, j / q}+\lambda \Delta t u_{l, j / q} & =u_{l,(j-1) / q} \\
u_{l, 0} & =(1+\alpha) u_{l-1,1} \\
u_{l, 1} & =(1+\alpha)\left(\frac{1}{1+\Delta t \lambda}\right)^{q} u_{l-1,1} .
\end{aligned}
$$

This implies that the stability functions of the implicit Euler method, used as the $\mathscr{G}$-propagator for the IDE (2) and the $\mathscr{F}$-propagator for the IDE (1), are $\mathscr{R}_{\mathrm{g}}(\Delta T \lambda)=(1+\beta) \mathscr{R}_{\text {Euler }}(\Delta T \lambda)$ and $\mathscr{R}_{f}(\Delta t \lambda)=(1+\alpha)^{p} \mathscr{R}_{\text {Euler }}^{p q}(\lambda \Delta t)$, respectively, i.e.,

$$
\begin{aligned}
\mathscr{R}_{\mathrm{g}}(z) & =(1+\beta) \mathscr{R}_{\text {Euler }}(z), \\
\mathscr{R}_{f}(z / J) & =(1+\alpha)^{p} \mathscr{R}_{\text {Euler }}^{J}(z / J) .
\end{aligned}
$$


From (10), $z=\Delta T \lambda$ and $\mathscr{R}_{\text {Euler }}(z)=1 /(1+z)$ is the stability function of the implicit Euler method applied to the regular ODE, $\alpha=0$ in (8).

For a general RK method used as the $\mathscr{F}$ propagator with the small step-size $\Delta t$, the function $\mathscr{R}_{f}(z / J)$ can be derived similarly,

$$
\mathscr{R}_{f}(z / J)=(1+\alpha)^{p} \mathscr{R}^{J}(z / J),
$$

where $\mathscr{R}(z)$ is the stability function of the RK method applied to (8) with $\alpha=0$. Particularly, for the Trapezoidal rule and the 4th-order Gauss4 method, the stability functions are

$$
\mathscr{R}(z)=\left\{\begin{array}{l}
\mathscr{R}_{\mathrm{TR}}(z)=\frac{1-\frac{z}{2}}{1+\frac{z}{2}}, \\
\mathscr{R}_{\text {Gauss } 4}(z)=\frac{1-\frac{z}{2}+\frac{z^{2}}{12}}{1+\frac{z}{2}+\frac{z^{2}}{12}} .
\end{array}\right.
$$

With the above preparations, the contraction functions (7) for the three parareal algorithms pararealEuler, parareal-TR, and parareal-Gauss4 are specified as

$$
\begin{aligned}
& \mathscr{K}_{\text {Euler,TR,Gauss } 4}(z, J)= \\
& \frac{\left|(1+\alpha)^{p} \mathscr{R}_{\text {Euler,TR,Gauss } 4}^{J}\left(\frac{z}{J}\right)-(1+\beta) \mathscr{R}_{\text {Euler }}(z)\right|}{1-\left|(1+\beta) \mathscr{R}_{\text {Euler }}(z)\right|} .
\end{aligned}
$$

By letting $\beta=(1+\alpha)^{p}-1$, we write

$$
\begin{aligned}
& \mathscr{K}_{\text {Euler,TR,Gauss } 4}(z, J) \\
& =\frac{\left|\mathscr{R}_{\text {Euler,TR,Gauss } 4}^{J}\left(\frac{z}{J}\right)-\mathscr{R}_{\text {Euler }}(z)\right|}{\gamma-\left|\mathscr{R}_{\text {Euler }}(z)\right|},
\end{aligned}
$$

with $\gamma=1 /|1+\alpha|^{p} \geqslant 1$. If $\beta \neq(1+\alpha)^{p}-1$, we cannot represent the contraction factor $\mathscr{K}_{\text {Euler,TR,Gauss } 4}(z, J)$ by the simple form (13), and the derivation of a sharp upper bound of $\mathscr{K}_{\text {Euler,TR,Gauss } 4}(z, J)$ is extremely complicated.

\section{CONVERGENCE ANALYSIS}

In this section, we derive a sharp upper bound of $\mathscr{K}_{\text {Euler,TR,Gauss } 4}(z, J)$, which depends only on $\gamma$. We obtain a function $\hat{\rho}(\gamma)$ such that $\max _{J \geqslant 2, z \geqslant 0} \mathscr{K}_{\text {Euler,TR,Gauss } 4}(z, J) \leqslant \hat{\rho}(\gamma)$.

\section{The Parareal-Euler algorithm}

We first consider the parareal-Euler algorithm, which consists of using the implicit Euler method for both the $\mathscr{G}$ - and $\mathscr{F}$-propagators.
Lemma 1 Let $\mathscr{R}_{\text {Euler }}(z)=1 /(1+z), z \geqslant 0$, be the stability function of the implicit Euler method and $J_{1}$, $J_{2}$ be two integers satisfying $J_{2}>J_{1} \geqslant 1$. Then

$$
\mathscr{R}_{\text {Euler }}^{J_{2}}\left(z / J_{2}\right) \leqslant \mathscr{R}_{\text {Euler }}^{J_{1}}\left(z / J_{1}\right)
$$

for all $z \geqslant 0$.

Proof: Let $s=z / J, r(s)=\mathscr{R}_{\text {Euler }}(s)$, and $R(s)=$ $\log r(s)-s r^{\prime}(s) / r(s)$. A routine calculation yields $\partial_{J}\left(\mathscr{R}_{\text {Euler }}^{J}(z / J)\right)=r^{J}(s) R(s)$. Clearly, it holds that $\operatorname{sign}\left(\partial_{J}\left(\mathscr{R}_{\text {Euler }}^{J}(z / J)\right)\right)=\operatorname{sign}(R(s))$. Then it suffices to prove $R(s) \leqslant 0$ for all $s \geqslant 0$. We have

$$
R^{\prime}(s)=-s \frac{\mathrm{d}^{2}}{\mathrm{~d} s^{2}}(\log r(s))=-\frac{s}{(1+s)^{2}} .
$$

Hence it holds that $R^{\prime}(s) \leqslant R(0)$ for $s \geqslant 0$. This together with $R(0)=0$ gives $R(s) \leqslant 0$, which implies $\partial_{J}\left(\mathscr{R}_{\text {Euler }}^{J}(z / J)\right) \leqslant 0$ for all $z \geqslant 0$.

From Lemma 1, we now present the sharp bound $\hat{\rho}(\gamma)$ of the convergence factor of the parareal-Euler algorithm.

Theorem 2 For any $\gamma=|1+\alpha|^{-p} \geqslant 1$, it holds that

$$
\max _{J \geqslant 2, z \geqslant 0} \mathscr{K}_{\text {Euler }}(z, J) \leqslant \hat{\rho}_{\text {Euler }}(\gamma)
$$

where

$$
\hat{\rho}_{\text {Euler }}(\gamma)=\frac{\gamma-\mathrm{e}^{-z^{\dagger}}}{\gamma-1 /\left(1+z^{\dagger}\right)}-1,
$$

with $z^{\dagger} \in(1,3)$ is the unique root of the equation $\gamma\left[(1+z)^{2}-\mathrm{e}^{z}\right]-z=0$.

Proof: By Lemma 1 we have

$$
\begin{aligned}
\mathscr{K}_{\text {Euler }}(z, J) & =\frac{\frac{1}{1+z}-\frac{1}{(1+z / J)^{J}}}{\gamma-\frac{1}{1+z}} \\
\max _{J \geqslant 2, z \geqslant 0}\left\{\mathscr{K}_{\text {Euler }}(z, J)\right\} & \leqslant \frac{\frac{1}{1+z}-\mathrm{e}^{-z}}{\gamma-\frac{1}{1+z}} \\
& =\frac{\gamma-\mathrm{e}^{-z}}{\gamma-\frac{1}{1+z}}-1=: \Phi(\gamma, z) .
\end{aligned}
$$

It holds that $\partial_{z} \Phi(\gamma, z)=\left(\left(\gamma\left[(1+z)^{2}-\mathrm{e}^{z}\right]-z\right) /(\gamma(1+\right.$ $\left.z)-1)^{2}\right) \mathrm{e}^{-z}$ and has a unique root $z^{\dagger}$, which is the global maximizer of $\Phi(\gamma, z)$ for $z \in[0, \infty)$. The root $z^{\dagger} \in(1,3)$ since $\partial_{z} \Phi(\gamma, 1)>0$ and $\partial_{z} \Phi(\gamma, 3)<0$.

\section{The Parareal-TR algorithm}

The stability function $\mathscr{R}_{\mathrm{TR}}(z)=(1-z / 2) /(1+z / 2)$ of the Trapezoidal rule has different signs for $z<2$ and $z>0$. Hence, the convergence properties of the parareal-TR algorithm consists of two cases, $J$ is even and $J$ is odd. We first consider the case $J$ is even, and the case for $J$ odd is commended in Remark 1. 
Lemma 2 Let $J \geqslant 2$ be an even integer. Then for any $\gamma \geqslant 1$,

$$
\mathscr{K}_{\mathrm{TR}}(z, J)= \begin{cases}\frac{\frac{1}{1+z}-\mathscr{R}_{\mathrm{TR}}^{J}\left(\frac{z}{J}\right)}{\gamma-\frac{1}{1+z}}, & z \in\left[0, J s_{0}\right], \\ \frac{\mathscr{R}_{\mathrm{TR}}^{J}\left(\frac{z}{J}\right)-\frac{1}{1+z}}{\gamma-\frac{1}{1+z}}, & z>J s_{0},\end{cases}
$$

where $s_{0} \geqslant 2$ is the unique root of the function $\phi(s, J)=J \log |(s-2) /(s+2)|+\log (1+J s)$.

Proof: Let $s=z / J$. Then $\mathscr{R}_{\mathrm{TR}}^{J}(z / J)-1 /(1+z)=$ $\mathscr{R}_{\mathrm{TR}}^{J}(s)-1 /(1+J s)$ and

$$
\operatorname{sign}\left(\mathscr{R}_{\mathrm{TR}}^{J}(z / J)-\frac{1}{1+z}\right)=\operatorname{sign}(\phi(s, J)) .
$$

Hence it suffices to check the sign of the function $\phi(s, J)$ with respect to $s$. We consider two cases, $s \in(0,2)$ and $s>2$. For $s \in(0,2)$, we have

$$
\partial_{J} \phi(s, J)=\log \frac{2-s}{s+2}+\frac{s}{1+J s}<\log \frac{2-s}{s+2}+s<0,
$$

which holds for any $J \geqslant 1$. Hence

$$
\phi(s, J) \leqslant \phi(s, 1)<\log \frac{2-s}{s+2}+s<0 .
$$

For $s>2, \phi(s, J)$ is an increasing function of $s$. This together with $\lim _{s \rightarrow 2^{+}} \phi(s, J)=-\infty$ and $\lim _{s \rightarrow \infty} \phi(s, J)=\infty$ implies that $\phi(s, J)$ has a unique root $s_{0} \geqslant 2$ with $\phi(s, J)<0$ for $s<s_{0}$ and $\phi(s, J)>0$ for $s>s_{0}$. The above analysis together with (17) gives (16).

Lemma 3 Let $\mathscr{R}_{\mathrm{TR}}(z)=(1-z / 2) /(1+z / 2)$ be the stability function of the Trapezoidal rule and $J^{*} \geqslant 2$. Then for any real numbers $J_{2}>J_{1} \geqslant J^{*}$,

$$
\mathscr{R}_{\mathrm{TR}}^{J_{2}}\left(z / J_{2}\right)>\mathscr{R}_{\mathrm{TR}}^{J_{1}}\left(z / J_{1}\right)
$$

for $z \in\left(0,2 J^{*}\right]$.

Proof: The partial derivative of $\mathscr{R}_{\mathrm{TR}}^{J}(z / J)$ with respect to $J$ leads to

$$
\begin{aligned}
& \partial_{J}\left[\mathscr{R}_{\mathrm{TR}}^{J}(z / J)\right]= \\
& \quad \mathscr{R}_{\mathrm{TR}}^{J}(z / J)\left[\log \mathscr{R}_{\mathrm{TR}}(z / J)-\frac{z}{J} \frac{\partial_{z} \mathscr{R}_{\mathrm{TR}}(z / J)}{\mathscr{R}_{\mathrm{TR}}(z / J)}\right] .
\end{aligned}
$$

Let $s=z / J, r(s)=\mathscr{R}_{\mathrm{TR}}(s)$ and $R(s)=\log r(s)-$ $s r^{\prime}(s) / r(s)$. Clearly, $\partial_{J}\left[\mathscr{R}_{\mathrm{TR}}^{J}(z / J)\right]=r^{J}(s) R(s)$. Since $z \in\left(0,2 J^{*}\right]$ and $J>J^{*}$, we have $s \in(0,2)$, which implies $r(s)=\mathscr{R}_{\mathrm{TR}}(z / J)>0$. Hence it suffices to prove $R(s)>0$ for $s \in(0,2)$. Note that

$$
R^{\prime}(s)=-s \frac{\mathrm{d}}{\mathrm{d} s}\left(\frac{r^{\prime}(s)}{r(s)}\right)=\frac{8 s^{2}}{\left(4-s^{2}\right)^{2}}>0 .
$$

Since $R(0)=0$, thus $R(s)>0$ for $s \in(0,2)$.

Based on Lemmas 2 and 3, we derive the following result for the upper bound of the contraction factor $\mathscr{K}_{\mathrm{TR}}(z, J)$ of the parareal-TR algorithm.

Theorem 3 Let $J \geqslant 2$ be an even integer. Then for any $\gamma=|1+\alpha|^{-p} \geqslant 1$ the contraction factor $\mathscr{K}_{\mathrm{TR}}(z, J)$ of the parareal-TR algorithm satisfies

$$
\max _{J \geqslant 2, z \geqslant 0}\left\{\mathscr{K}_{\mathrm{TR}}(z, J)\right\} \leqslant \hat{\rho}_{\mathrm{TR}}(\gamma),
$$

where

$$
\begin{aligned}
& \hat{\rho}_{\mathrm{TR}}(\gamma)=\max \left\{\varrho(\gamma), \frac{1}{\gamma}\right\}, \\
& \varrho(\gamma)=\max _{z \in[0,4]} \frac{1-(1+z)\left(\left(1-\frac{z}{4}\right) /\left(1+\frac{z}{4}\right)\right)^{2}}{\gamma(1+z)-1} .
\end{aligned}
$$

Proof: The proof consists of two parts.

Part A: $\max _{z \in\left[0, J s_{0}\right]} \mathscr{K}_{\mathrm{TR}}(z, J) \leqslant \varrho(\gamma)$ for all even integers $J \geqslant 2$, where $s_{0} \geqslant 2$ is the quantity defined in Lemma 2. To prove this, we set an ansatz $\max _{z \in\left[0, J s_{0}\right]} \mathscr{K}_{\mathrm{TR}}(z, J) \leqslant \theta$ with $\theta \geqslant 1 /(5 \gamma-1)$, and prove that $\theta$ can reach $\varrho(\gamma)$. By Lemma 2, $\mathscr{K}_{\mathrm{TR}}(z, J) \leqslant \theta$ is equivalent to

$$
\frac{1+\theta(1-\gamma)-\theta \gamma z}{1+z} \leqslant \mathscr{R}_{\mathrm{TR}}^{J}\left(\frac{z}{J}\right)
$$

for all $z \in\left[0, J s_{0}\right]$. Since $\theta \geqslant 1 /(5 \gamma-1)$, it suffices to prove that (19) holds for $z \in[0,4]$ as for $z>4$,

$$
1+\theta(1-\gamma)-\theta \gamma z<1-\theta(5 \gamma-1) \leqslant 0 .
$$

For $z \in[0,4]$ and $J \geqslant 2$, by Lemma 3 we have $\mathscr{R}_{\mathrm{TR}}^{J}(z / J) \geqslant \mathscr{R}_{\mathrm{TR}}^{2}(z / 2)=(1-z / 4)^{2} /(1+z / 4)^{2}$. Hence, to guarantee (19), it suffices to require that $\mathscr{R}_{\mathrm{TR}}^{2}(z / 2) \geqslant(1+\theta(1-\gamma)-\theta \gamma z) /(1+z)$, i.e.,

$$
\theta \geqslant \max _{z \in[0,4]} \frac{1-(1+z) \mathscr{R}_{\mathrm{TR}}^{2}\left(\frac{z}{2}\right)}{\gamma(1+z)-1}=\varrho(\gamma) .
$$

Hence $\varrho(\gamma)$ is a reliable upper bound of $\mathscr{K}_{\mathrm{TR}}(z, J)$ for $z \in\left[0, J s_{0}\right]$.

Part B: $\max _{z \geqslant J s_{0}} \mathscr{K}_{\mathrm{TR}}(z, J)=1 / \gamma$. Since $J s_{0}>2 J$ and for $z>2 J$, it holds that $\mathscr{R}_{\mathrm{TR}}(z / J)<0$, and from Lemma 2,

$$
\mathscr{K}_{\mathrm{TR}}(z, J)=\frac{\left(-\mathscr{R}_{\mathrm{TR}}(z / J)\right)^{J}-1 /(1+z)}{1-1 /(1+z)}
$$


for $z>J s_{0}$. Hence

$$
\begin{aligned}
& (\gamma(1+z)-1)^{2} \partial_{z} \mathscr{K}_{\mathrm{TR}}(z, J) \\
& =\left(-\mathscr{R}_{\mathrm{TR}}(z / J)\right)^{J-1} \frac{4(\gamma(1+z)-1)(1+z)}{(2+z / J)^{2}} \\
& +\gamma-\left(-\mathscr{R}_{\mathrm{TR}}(z / J)\right)^{J}>0 .
\end{aligned}
$$

This implies $\partial_{z} \mathscr{K}_{\mathrm{TR}}(z, J)>0$ for $z>J s_{0}$. Hence,

$$
\max _{z \geqslant J s_{0}} \mathscr{K}_{\mathrm{TR}}(z, J)=\lim _{z \rightarrow \infty} \mathscr{K}_{\mathrm{TR}}(z, J)=\frac{1}{\gamma},
$$

since $\mathscr{K}_{\mathrm{TR}}(z, J)=\frac{\mathscr{R}_{\mathrm{TR}}^{J}(z / J)-1 /(1+z)}{\gamma-1 /(1+z)}$ and $\lim _{z \rightarrow \infty} \mathscr{R}_{\mathrm{TR}}(z)=$ -1 .

Remark 1 Theorem 3 also holds for an odd integer $J \geqslant 3$. From the proof of Lemma 2 for all $z \geqslant 0$,

$$
\mathscr{K}_{\mathrm{TR}}(z, J)=\frac{\frac{1}{1+z}-\mathscr{R}_{\mathrm{TR}}^{J}\left(\frac{z}{J}\right)}{\gamma-\frac{1}{1+z}} .
$$

Hence, similar to (20), we have

$$
\begin{aligned}
& (\gamma(1+z)-1)^{2} \partial_{z} \mathscr{K}_{\mathrm{TR}}(z, J) \\
& \quad=\mathscr{R}_{\mathrm{TR}}^{J-1}\left(\frac{z}{J}\right) \frac{4(\gamma(1+z)-1)(1+z)}{\left(2+\frac{z}{J}\right)^{2}}+\mathscr{R}_{\mathrm{TR}}^{J}\left(\frac{z}{J}\right)-\gamma \\
& \geqslant \mathscr{R}_{\mathrm{TR}}^{J-1}\left(\frac{z}{J}\right) \frac{4(\gamma(1+z)-1)(1+z)}{\left(2+\frac{z}{J}\right)^{2}}-1-\gamma .
\end{aligned}
$$

Since $J$ is an odd integer, it is easy to verify that $\mathscr{R}_{\mathrm{TR}}^{J-1}(z / J)(4(\gamma(1+z)-1)(1+z)) /(2+z / J)^{2}$ is an increasing function of $z$ for $z>2 J$. Furthermore, since

$$
\lim _{z \rightarrow \infty} \mathscr{R}_{\mathrm{TR}}^{J-1}\left(\frac{z}{J}\right) \frac{4(\gamma(1+z)-1)(1+z)}{\left(2+\frac{z}{J}\right)^{2}}=4 \gamma J^{2},
$$

for given $J \geqslant 3$ and $\gamma \geqslant 1$, we can always choose some quantity $s_{0} \geqslant 2$ such that

$$
\mathscr{R}_{\mathrm{TR}}^{J-1}\left(\frac{z}{J}\right) \frac{4(\gamma(1+z)-1)(1+z)}{\left(2+\frac{z}{J}\right)^{2}}>1+\gamma,
$$

for $z \geqslant J s_{0}$. With such $s_{0},(21)$ implies $\partial_{z} \mathscr{K}_{\mathrm{TR}}(z, J)>$ 0 for $z \geqslant J s_{0}$. Thus from the proofs of Part A and Part $B$ given above, it is easy to see that Theorem 3 is also applicable to an odd integer $J \geqslant 3$.

\section{The Parareal-Gauss4 algorithm}

It remains to consider the parareal-Gauss 4 algorithm, which consists of using the 4th-order Gauss RK method for the $\mathscr{F}$-propagator. The converged solution of the parareal-Gauss 4 algorithm is of order $4, u_{n}^{\infty}-u\left(T_{n}\right)=O\left(\Delta t^{4}\right)$.
Lemma 4 Let $\mathscr{R}_{\text {Gauss } 4}(z)=\left(1-z / 2+z^{2} / 12\right) /(1+$ $\left.z / 2+z^{2} / 12\right)$. Then, for $J_{2}>J_{1} \geqslant 2$ it holds that, for all $z>0$,

$$
\mathscr{R}_{\text {Gauss } 4}^{J_{2}}\left(z / J_{2}\right)<\mathscr{R}_{\text {Gauss } 4}^{J_{1}}\left(z / J_{1}\right) .
$$

Proof: Let $s=z / J, r(s)=\mathscr{R}_{\text {Gauss } 4}(s)$, and $R(s)=$ $\log r(s)-s r^{\prime}(s) / r(s)$. Then for $z>0$ and $J \geqslant 2$,

$$
\begin{aligned}
& \operatorname{sign}\left(\partial_{J} \mathscr{R}_{\text {Gauss } 4}^{J}(z / J)\right)=\operatorname{sign}(R(s)), \\
& R^{\prime}(s)=\frac{24 s^{4}\left(s^{2}-24\right)}{\left[\left(1-6 z+z^{2}\right)\left(1+6 z+z^{2}\right)\right]^{2}} .
\end{aligned}
$$

This gives $\max _{s \geqslant 0} R(s)=\max \left\{R(0), \lim _{s \rightarrow \infty} R(s)\right\}=$ 0 , since $r(0)=\lim _{s \rightarrow \infty} r(s)=1, r^{\prime}(0)=-1$, and $\lim _{s \rightarrow \infty} r^{\prime}(s)=0$. Hence, it holds that $\partial_{J} \mathscr{R}_{\text {Gauss } 4}^{J}<0$ for any $J \geqslant 2$, which proves (22).

Theorem 4 Let $\gamma=|1+\alpha|^{-p} \geqslant 1$ and $J \geqslant 2$ be an integer. Then, for the parareal-Gauss 4 algorithm, the contraction factor $\mathscr{K}_{\text {Gauss } 4}(z, J)$ defined by (13) satisfies $\max _{J \geqslant 2, z \geqslant 0} \mathscr{K}_{\mathrm{TR}}(z, J) \leqslant \hat{\rho}_{\text {Gauss } 4}(\gamma)$, where

$$
\begin{array}{r}
\hat{\rho}_{\text {Gauss } 4}(\gamma)=\max \left\{\varrho(\gamma), \frac{1}{\gamma}\right\}, \\
\varrho(\gamma)=\frac{1-\left(1+z^{\dagger}\right) \mathrm{e}^{-z^{\dagger}}}{\gamma\left(1+z^{\dagger}\right)-1},
\end{array}
$$

and $z^{\dagger} \in(1,3)$ is the unique root of the equation $\gamma\left[(1+z)^{2}-\mathrm{e}^{z}\right]-z=0$.

Proof: The proof is similar to that of Theorem 3 concerning the parareal-TR algorithm. Precisely, following the proof of Lemma 2, we fix some quantity $s_{0} \geqslant 4$ such that

$$
\mathscr{K}_{\text {Gauss } 4}(z, J)= \begin{cases}\frac{\frac{1}{1+z}-\mathscr{R}_{\text {Gauss } 4}^{J}\left(\frac{z}{J}\right)}{\gamma-\frac{1}{1+z}}, & z \in\left[0, J s_{0}\right], \\ \frac{\mathscr{R}_{\text {Gauss } 4}^{J}\left(\frac{z}{J}\right)-\frac{1}{1+z}}{\gamma-\frac{1}{1+z}}, & z>J s_{0} .\end{cases}
$$

For the first interval $z \in\left[0, J s_{0}\right]$, we claim that $\max _{J \geqslant 2, z \in\left[0, J s_{0}\right]} \mathscr{K}_{\text {Gauss } 4}(z, J) \leqslant \varrho(\gamma)$. As with Part A in the proof of Theorem 3, we set an ansatz $\max _{z \in\left[0, J s_{0}\right]} \mathscr{K}_{\text {Gauss } 4}(z, J) \leqslant \theta$ with $\theta \geqslant 1 /(5 \gamma-1)$, and prove that $\theta$ can reach $\varrho(\gamma)$. As with (19), we have

$$
\begin{aligned}
\mathscr{K}_{\text {Gauss } 4}(z, J) & \leqslant \theta \\
\frac{1+\theta(1-\gamma)-\theta \gamma z}{1+z} & \leqslant \mathscr{R}_{\text {Gauss } 4}^{J}\left(\frac{z}{J}\right),
\end{aligned}
$$

for all $z \in\left[0, J s_{0}\right]$. 
By the assumption $\theta \geqslant 1 /(5 \gamma-1)$, it suffices to prove (24) for $z \in[0,4]$, since for $z>4$ we have $1+\theta(1-\gamma)-\theta \gamma z<1-\theta(5 \gamma-1) \leqslant 0$. For $z \in[0,4]$ and $J \geqslant 2$, Lemma 4 gives $\mathscr{R}_{\text {Gauss } 4}^{J}(z / J) \geqslant$ $\lim _{J \rightarrow \infty} \mathscr{R}_{\text {Gauss } 4}^{J}=\mathrm{e}^{-z}$. Hence, it suffices to have $\mathrm{e}^{-z} \geqslant(1+\theta(1-\gamma)-\theta \gamma z) /(1+z)$ to guarantee (24), i.e.,

$$
\theta \geqslant \max _{z \in[0,4]} \frac{1-(1+z) \mathrm{e}^{-z}}{\gamma(1+z)-1}=\varrho(\gamma) .
$$

Hence $\varrho(\gamma)$ is a reliable upper bound of $\mathscr{K}_{\mathrm{TR}}(z, J)$ for $z \in\left[0, J s_{0}\right]$. For a given $\gamma \geqslant 1$, the function $\left(1-(1+z) \mathrm{e}^{-z}\right) /(\gamma(1+z)-1)$ attains its maximum at $z=z^{\dagger}$, which is already proved in the proof of Theorem 2 .

Note that, for $z \in\left[J s_{0}, \infty\right)$,

$$
\begin{aligned}
& {[\gamma(1+z)-1]^{2} \partial_{z} \mathscr{K}_{\text {Gauss } 4}(z, J)=} \\
& \mathscr{R}_{\text {Gauss } 4}^{J-1}\left(\frac{z}{J}\right) \frac{[\gamma(1+z)-1](1+z)\left(\frac{z^{2}}{12 J^{2}}-1\right)}{\left(1+\frac{z}{2 J}+\frac{z^{2}}{12 J^{2}}\right)^{2}} \\
& +1-\mathscr{R}_{\text {Gauss } 4}^{J}(z / J)
\end{aligned}
$$

and $s_{0}>4$, implying $J^{2} s_{0}^{2}>12 J^{2}$, thus we have $\partial_{z} \mathscr{K}_{\text {Gauss } 4}(z, J)>0$ for $z \geqslant J s_{0}$. Note that for the 4th-order Gauss RK method, for $z>0$, it holds that $\mathscr{R}_{\text {Gauss }}^{J}(z) \in(0,1)$ for both even and odd $J$. Hence,

$$
\max _{z \geqslant J s_{0}} \mathscr{K}_{\text {Gauss } 4}(z, J) \leqslant \lim _{z \rightarrow \infty} \mathscr{K}_{\text {Gauss } 4}(z, J)=\frac{1}{\gamma} .
$$

\section{Discussions}

We now discuss the upper bounds $\hat{\rho}_{\text {Euler,TR,Gauss } 4}(\gamma)$ of the three parareal algorithms. With $\gamma=|1+\alpha|^{-p}$ and four values of $p$, Fig. 1 shows the profile of the three bounds as functions of $\alpha \in[-2,0]$. For a given $\gamma$, the values of $\hat{\rho}_{\mathrm{TR}}(\gamma)$ and $\hat{\rho}_{\text {Gauss } 4}(\gamma)$ are very close, as shown together in Fig. 1a.

For a given impulsive parameter $\alpha \in(-2,0)$, all three parareal algorithms possess a constant convergence factor less than 1 , and the constant convergence factor becomes smaller when $p$ is larger (Fig. 1), i.e., a single large step-size $\Delta T$ contains more impulses. Furthermore, for a given $\gamma$ the parareal-Euler algorithm has smaller convergence factor than the other two algorithms.

One of particular interest is the behaviour of $\hat{\rho}_{\text {Euler,TR,Gauss } 4}(\gamma)$ as $\gamma$ approaches 0 and 1 , which, respectively, correspond to $\alpha=-1$ and $\alpha=-2$ (or $\alpha=0$ ).

For $\alpha=-1, \hat{\rho}_{\text {Euler,TR,Gauss } 4}=0$, which implies that all three algorithms converge in one iteration.
For $\alpha=-1, \Delta u\left(\tau_{l}\right)=\alpha u\left(\tau_{l}\right)$, which is equivalent to $u\left(\tau_{l}^{+}\right)=0$, and this implies that the IDE is a completely autonomic differential equation in the subinterval $\left[\tau_{l}, \tau_{l+1}\right]$ with the zero initial value. Hence we are solving a series of independent ODEs on the time intervals $\left\{\left[\tau_{l}, \tau_{l+1}\right]\right\}_{l \geqslant 0}$ with $\tau_{0}=0$ and $\tau_{l+1}-\tau_{l}=\tau$ by the parareal algorithm. Since $\Delta T \geqslant \tau$, we have only one large subinterval in the framework of the parareal algorithm. Hence we obtain the converged solution after one iteration. This can also be explained by using the property of convergence in finite iterations for the parareal algorithm ${ }^{15}$.

For $\alpha=-2$ or $\alpha=0$, i.e., $\gamma=1$, we have $\hat{\rho}_{\mathrm{TR}, \mathrm{Gauss} 4}=1$ and $\hat{\rho}_{\text {Euler }}=0.3$. For $\gamma=1$, the contraction factors $\mathscr{K}_{\text {Euler,TR,Gauss } 4}(z, J)$ is reduced to

$$
\mathscr{K}_{\text {Euler,TR,Gauss } 4}(z, J)=\frac{\left|\mathscr{R}_{\text {Euler,TR,Gauss } 4}^{J}\left(\frac{z}{J}\right)-\frac{1}{1+z}\right|}{1-\frac{1}{1+z}},
$$

which is exactly the contraction factors of the three parareal algorithms applied to the regular ODEs without impulses ${ }^{15}$. In this case, from Ref. 16 we have $\max _{J \geqslant 2, z \geqslant 0} \mathscr{K}_{\text {Euler }}(z, J)=0.298$, which coincides with the message implied by Fig. 1b for $\alpha=0$ and $\alpha=-2$. For the parareal-TR and pararealGauss4 algorithms, we have

$$
\begin{aligned}
& \lim _{z \rightarrow \infty} \mathscr{K}_{\mathrm{TR}, \mathrm{Gauss} 4}(z, J) \\
&=\lim _{z \rightarrow \infty} \frac{\left|\mathscr{R}_{\mathrm{TR}, \mathrm{Gauss} 4}^{J}\left(\frac{z}{J}\right)-\frac{1}{1+z}\right|}{1-\frac{1}{1+z}}=1,
\end{aligned}
$$

since $\lim _{z \rightarrow \infty}\left|\mathscr{R}_{\mathrm{TR}, \text { Gauss } 4}^{J}(z / J)\right|=1$. This perfectly explains what we have observed in Fig. 1a for $\alpha=0$ and $\alpha=-2$.

\section{NUMERICAL EXPERIMENTS}

In this section, we provide numerical results to validate the theoretical prediction given in previous sections. We first consider a linear problem arising from semi-discretizing the impulsive heat equations and then consider the impulsive logistic equation, a nonlinear problem arising from population dynamics $^{25}$.

\section{Impulsive heat equations}

Consider the following impulsive heat equations

$$
\begin{aligned}
& u_{t}-u_{x x}=2 t \sin \left(4(1-x) t+t^{2} x\right), \\
&(x, t) \in(0,1) \times(0, T), t \neq \tau_{l}, \\
& \Delta u=\alpha u, \quad(x, t) \in(0,1) \times\left\{\tau_{l}\right\}_{l} \geqslant 1
\end{aligned}
$$



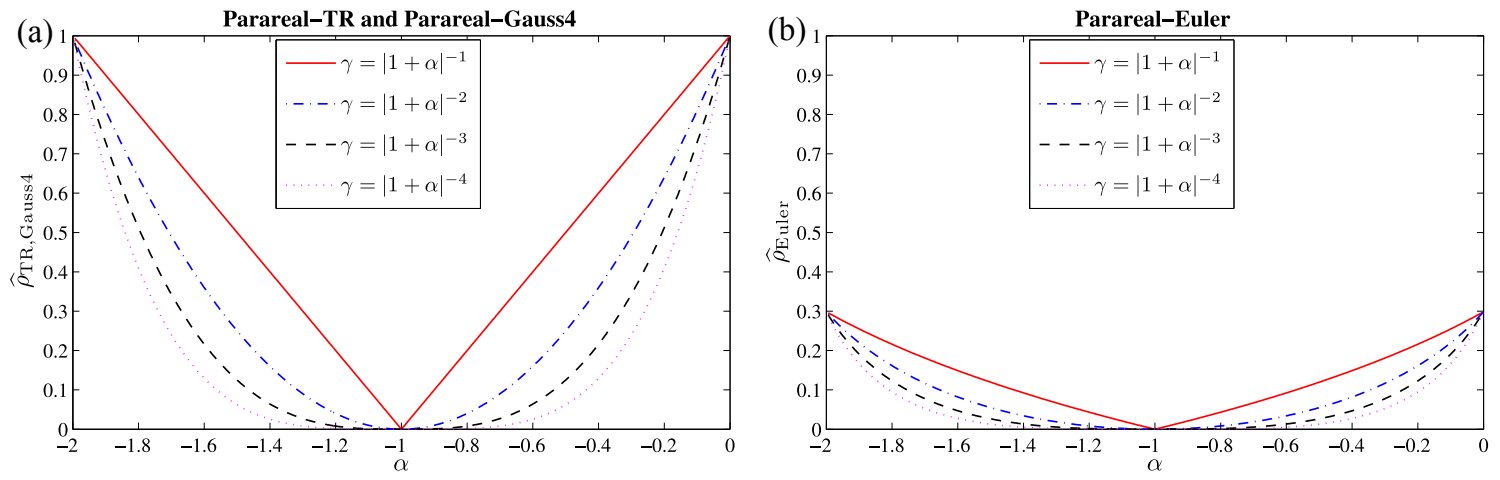

Fig. 1 The upper bounds $\hat{\rho}_{\text {Euler,TR,Gauss } 4}(\gamma)$ with $\gamma=|1+\alpha|^{-p}$ as a function of $\alpha \in(-2,0)$.

with $u(0, t)=u(1, t)=0$ and $u(x, 0)=0$. By using the centred finite difference formula with mesh size $\Delta x$ to discretize $\partial_{x}^{2}$, we obtain the ODEs system:

$$
\begin{aligned}
\mathbf{u}^{\prime}(t)+A \mathbf{u}(t) & =\mathbf{f}(t), & & t \neq \tau_{l}, \\
\Delta \mathbf{u}(t) & =\alpha \mathbf{u}(t), & & t=\tau_{l}, \\
\mathbf{u}(0) & =0, & &
\end{aligned}
$$

where $\mathbf{u}(t)=\left(u_{1}(t), u_{2}(t), \ldots, u_{m}(t)\right)^{T}$ with $u_{j}(t) \approx$ $u\left(x_{j}, t\right), x_{j}=j \Delta x$, and $j=1,2, \ldots, m=1 / \Delta x-1$. The matrix $A$ and the source term $\mathbf{f}(t)$ are

$$
\begin{aligned}
& A=\frac{1}{\Delta x^{2}}\left[\begin{array}{ccccc}
2 & -1 & & & \\
-1 & 2 & -1 & & \\
& \ddots & \ddots & \ddots & \\
& & -1 & 2 & -1 \\
& & & -1 & 2
\end{array}\right]_{m \times m} \\
& f(t)=2 t\left[\begin{array}{c}
\sin \left(4\left(1-x_{1}\right) t+t^{2} x_{1}\right) \\
\sin \left(4\left(1-x_{2}\right) t+t^{2} x_{2}\right) \\
\vdots \\
\sin \left(4\left(1-x_{m}\right) t+t^{2} x_{m}\right)
\end{array}\right] \text {. }
\end{aligned}
$$

Let $\Delta x=0.01, T=20, \tau=1 / 3, \Delta T=p \tau, \alpha=$ -1.45 , and $\Delta t=\Delta T / J$ with $J=20$. Fig. 2 shows the measured error $\max _{1 \leqslant n \leqslant N}\left\|\mathbf{u}_{n}^{k}-\mathbf{u}_{n}\right\|_{\infty}$ at each iteration for the three parareal algorithms, where $\left\{\mathbf{u}_{n}\right\}_{n=1}^{N}$ with $N=T / \Delta T$ denotes the converged solution. We consider two values of $p, p=1$ (Fig. 2a) and $p=2$ (Fig. 2b). The error predicted by the linear bound $\hat{\rho}_{\text {Euler,TR,Gauss } 4}$, i.e., $\max _{1 \leqslant n \leqslant N} \| \mathbf{u}_{n}^{0}-$ $\mathbf{u}_{n} \|_{\infty} \hat{\rho}_{\text {Euler,TR,Gauss }}^{k}(\gamma)$ with $\gamma=|1+\alpha|^{-p}$, is shown by the dotted lines. As already mentioned, for a given $\gamma$ the quantities $\hat{\rho}_{\mathrm{TR}}(\gamma)$ and $\hat{\rho}_{\text {Gauss }}(\gamma)$ are very close, there is only one dotted line for the pararealTR and parareal-Gauss4 algorithms in Fig. 2.

Fig. 2 shows that linear bounds for the three algorithms are sharp, and for a given impulsive parameter $\alpha$, all three parareal algorithms converge faster when a single large step-size $\Delta T$ contains more impulses. These observations coincide with the theoretical predictions implied by Fig. 1. We now validate the robustness of the convergence rates of the three parareal algorithms with respect the change of space and time discretization parameters. We show in Fig. 3 the effects of the space discretization parameter $\Delta x$ (Fig. 3a) and the ratio $J=\Delta T / \Delta t$ (Fig. 3b) on the convergence rates of the three parareal algorithms. For given $\Delta x$ (or $J$ ) we show the iteration number needed to reach the error tolerance $10^{-12}$, i.e., $\max _{1 \leqslant n \leqslant N}\left\|\mathbf{u}_{n}^{k}-\mathbf{u}_{n}\right\|_{\infty} \leqslant$ $10^{-12}$. Fig. 3 implies that the three parareal algorithms possess strongly robust convergence rates with respect to the reduction of $\Delta x$ and the increase of $J$. Particularly, it is interesting to point out that the parareal-TR and parareal-Gauss4 algorithms converge faster when a larger $J$ is used (Fig. 3b). This means that increasing the ratio $J$ to improve the accuracy of the converged solution does not deteriorate the convergence rates in practice.

\section{Impulsive logistic equation}

Consider a nonlinear problem, the impulsive logistic equation $^{25}$,

$$
\begin{gathered}
u_{t}-\left(u_{x x}+u_{y y}\right)-a u[K-u]=0, \\
(x, y) \in \Omega, t \in(0, T), t \neq \tau_{l}, \\
\Delta u=\alpha u(x, y, t), \quad(x, y) \in \Omega, t \in\left\{\tau_{l}\right\}_{l} \geqslant 1
\end{gathered}
$$

where $\Omega=(0,1)^{2}, a>0$, and $K>0$. We consider the Dirichlet boundary condition $u(x, y, t)=$ 0 for $(x, y) \in \partial \Omega$ and constant initial condition $u(x, y, 0)=1$ for $(x, y) \in \Omega$. Applying the centred finite difference formula to the spatial derivatives in 

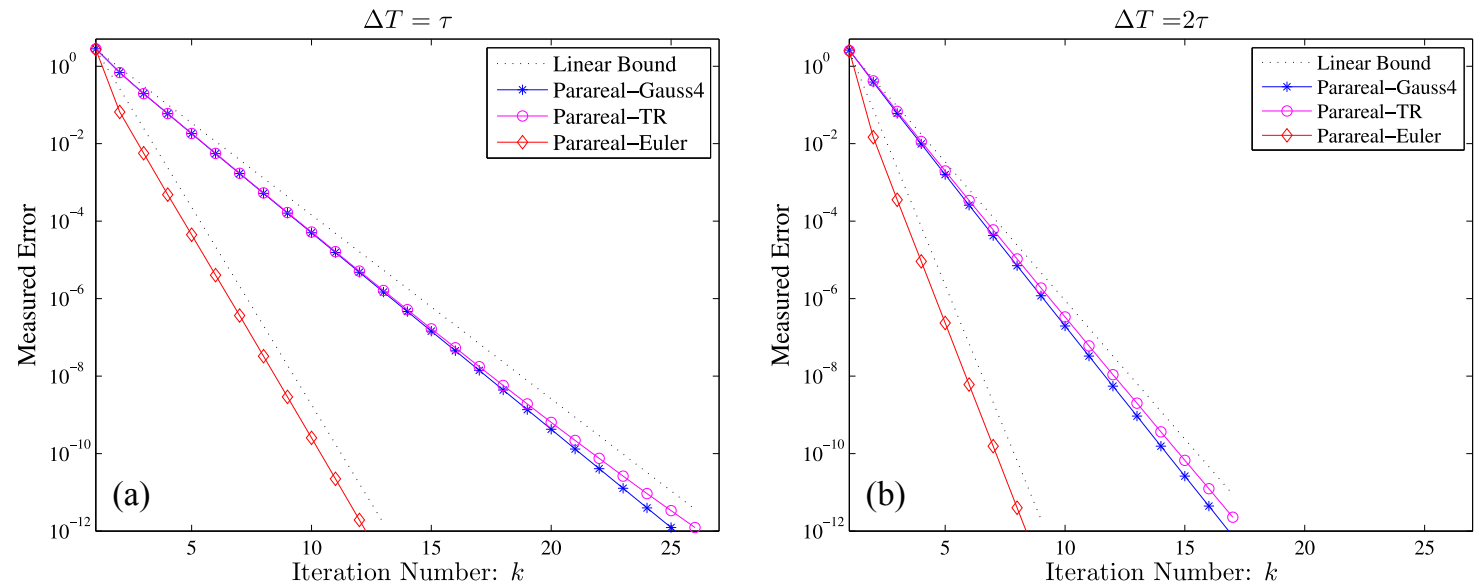

Fig. 2 Measured errors of the three parareal algorithms at each iteration, together with the error predicted by the linear bound, $\max _{1 \leqslant n \leqslant N}\left\|\mathbf{u}_{n}^{0}-\mathbf{u}_{n}\right\|_{\infty} \hat{\rho}_{\text {Euler,TR,Gauss }}^{k}$, for $\Delta T=p \tau$; (a) $p=1$, (b) $p=2$.
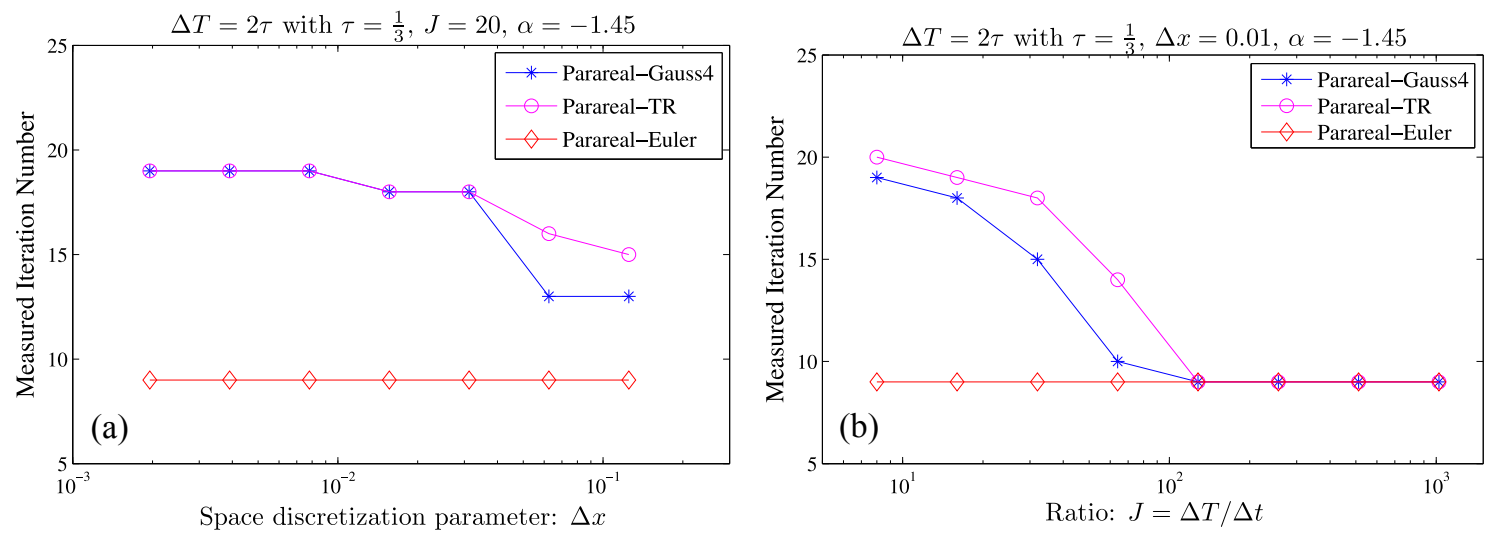

Fig. 3 Verification of the robustness of the convergence rates of the three parareal algorithms with respect to the change of (a) the space discretization parameter $\Delta x$ and (b) the ratio $J=\Delta T / \Delta t$.

(28), we obtain the impulsive ODEs:

$$
\begin{cases}\mathbf{u}^{\prime}(t)+\operatorname{Au}(t)-a \mathbf{u}(t) \circ[K \mathbf{e}-\mathbf{u}(t)]=0, & t \neq \tau_{l}, \\ \Delta \mathbf{u}(t)=\alpha \mathbf{u}(t), & t=\tau_{l}, \\ \mathbf{u}(0)=1, & \end{cases}
$$

where $\mathbf{u} \in \mathbb{R}^{m^{2}}, \mathbf{e}=(1,1, \ldots, 1) \in \mathbb{R}^{m^{2}}, \mathbf{A}=A \otimes I+I \otimes$ $A$ with $m=1 / \Delta x-1$, the identity matrix $I \in \mathbb{R}^{m \times m}$, and $A$ is the matrix defined by (27). The symbols $\otimes$ and $\circ$, respectively, denote the Kronecker product and Hadamard product; for any $u, v \in \mathbb{R}^{l}, u \circ v=$ $\left(u_{1} v_{1}, u_{2} v_{2}, \ldots, u_{l} v_{l}\right)$. Here, we discretize the spatial derivatives $\partial_{x}^{2}$ and $\partial_{y}^{2}$ by the same mesh size $\Delta x$.

We choose $a=0.2, K=2, \alpha=-0.7, \tau=0.4$, and $T=40$ for the problem parameters. With $\Delta T=2 \tau$ and $J=50$, we show in Fig. 4a the iteration number needed to reach the error tolerance
$10^{-12}$ for $\Delta x$ varies from large to small. Similar information for the case that $\Delta x=0.02$ is fixed and the ratio $J=\Delta T / \Delta t$ varies from small to large is shown in Fig. $4 \mathrm{~b}$. Clearly, similar to the linear case, the three parareal algorithms analysed in this paper still possess strongly robust convergence rates for nonlinear problems, with respect to reduction of the spatial discretization parameter $\Delta x$ and the increase of the ratio $J=\Delta T / \Delta t$.

\section{CONCLUSIONS}

We have analysed the convergence properties of the parareal algorithm for differential equation with impulses. The parareal algorithm studied here consists of using for the $\mathscr{G}$-propagator the implicit Euler method and for the $\mathscr{F}$-propagator three representative Runge-Kutta methods, the implicit Euler method, the Trapezoidal rule and the 4th-order 

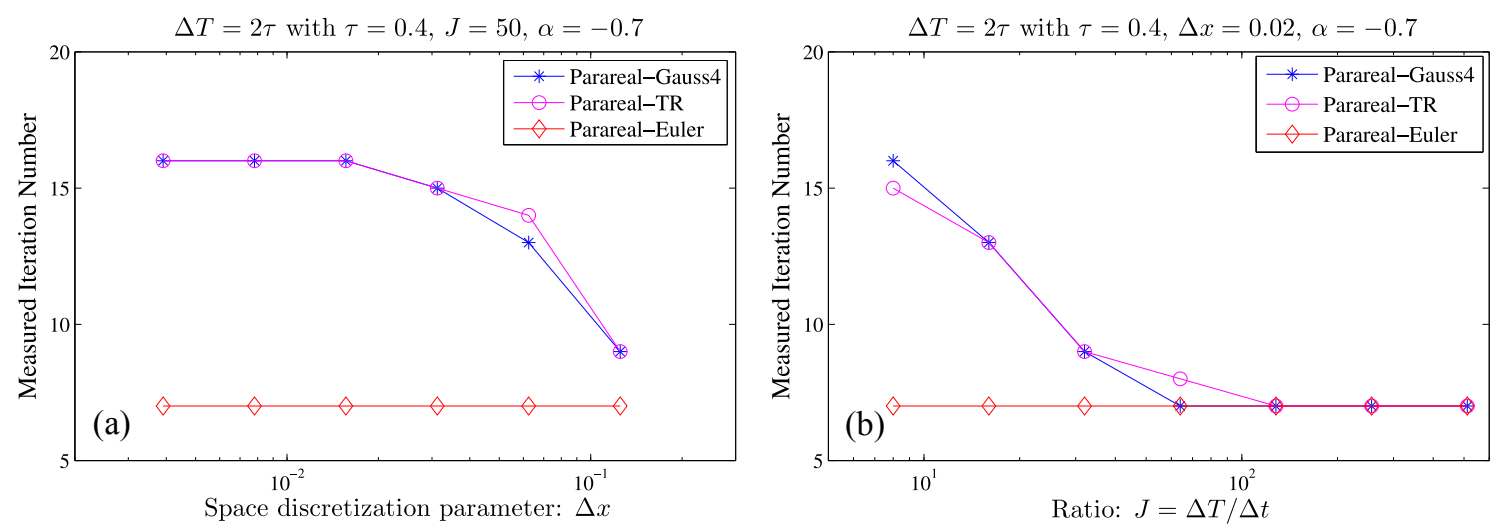

Fig. 4 For the nonlinear impulsive IDE (29) arising from semi-discretizing the impulsive logistic equation (28), the three parareal algorithms still possess robust convergence rates with respect to reduction of (a) the spatial discretization parameter $\Delta x$ and (b) the increase of the ratio $J=\Delta T / \Delta t$.

Gauss Runge-Kutta method. The converged solution of the resulting three parareal algorithms is of order one, two and four, respectively. The effect of the impulses on the convergence rate of the parareal algorithm is characterized by two parameters, the impulsive magnitude $\alpha$ and the impulsive frequency $p$ within one large step-size (i.e., $\Delta T=p \tau$ ). With given $\alpha$ and $p$, the $\mathscr{G}$-propagator is applied to a modified impulsive differential equations. We performed a rigorous convergence analysis for the three parareal algorithms, and the dependence of the convergence rate on the impulsive parameters is derived. An interesting finding is that when the impulses are of stable type, i.e., $\alpha \in(-2,0)$, we can expect faster convergence for the three parareal algorithms and particularly for the case $\mathscr{F}=$ Trapezoidal rule the parareal algorithm also possesses a constant convergence factor $\rho<1$, which is independent of the ratio $J=\Delta T / \Delta t$ and the eigenvalue distribution of the coefficient matrix $A$. This result is interesting because it is well known that for the regular differential equation (without impulses), the convergence factor of this parareal algorithm approaches 1 when $\lambda_{\max } \rightarrow \infty$, where $\lambda_{\max }$ denotes the largest eigenvalue of the coefficient matrix.

Acknowledgements: The authors are very grateful to the anonymous referees for their careful reading of a preliminary version of the manuscript and their valuable suggestions and comments, which greatly improved the quality of this paper. This work is supported by NSFC (11771313), the Project of China Postdoctoral (2015M580777, 2016T90841), the NSF of Science and Technology Department of
Sichuan Province (2018JY0469) and the NSF of SUSE (2015LX01,2016QZJ02).

\section{REFERENCES}

1. Mil'Man VD, Myshkis AD (1960) On the stability of motion in the presence of impulses. Sib Math $J$ 1, 233-7.

2. Samoilenko AM, Perestyuk NA, Chapovsky Y (1995) Impulsive Differential Equations, World Scientific, Singapore.

3. Bainov D, Simeonov $\mathrm{P}(1989)$ Systems with Impulsive Effect: Stability, Theory and Applications, Ellis Horwood, Chichester.

4. Bainov D, Simeonov $\mathrm{P}(1993)$ Impulsive Differential Equations: Periodic Solutions and Applications, Chapman \& Hall/CRC.

5. Akhmet MU (2003) On the general problem of stability for impulsive differential equations. $J$ Math Anal Appl 288, 182-96.

6. Benchohra M, Henderson J, Ntouyas S (2006) Impulsive Differential Equations and Inclusions, Hindawi Publishing Corporation, New York.

7. Stamov GT (2012) Almost Periodic Solutions of Impulsive Differential Equations, Springer Science \& Business Media.

8. Ran XJ, Liu MZ, Zhu QY (2008) Numerical methods for impulsive differential equation. Math Comput Modelling 48, 46-55.

9. Din Q, Donchev T, Nosheen A, Rafaqat M (2015) Runge-Kutta methods for differential equations with variable time of impulses. Numer Func Anal Opt 36, 777-91.

10. Liu X, Zhang GL, Liu MZ (2014) Analytic and numerical exponential asymptotic stability of nonlinear impulsive differential equations. Appl Numer Math 81, 40-9.

11. Liang H (2017) $h p$-Legendre-Gauss collocation 
method for impulsive differential equations. Int $J$ Comput Math 94, 151-72.

12. Lions JL, Maday Y, Turinici G (2001) A parareal in time discretization of PDE's. CR Acad Sci Paris Sér I Math 332, 661-8.

13. Emmett M, Minion ML (2012) Toward an efficient parallel in time method for partial differential equations. Comm Appl Math Comput Sci 7, 105-32.

14. Minion M, Speck R, Bolten M, Emmett M, Ruprecht D (2015) Interweaving PFASST and parallel multigrid. SIAM J Sci Comput 37, 244-63.

15. Gander MJ, Vandewalle S (2007) Analysis of the parareal time-parallel time-integration method. SIAM J Sci Comput 29, 556-78.

16. Mathew TR, Sarkis M, Schaerer CE (2010) Analysis of block parareal preconditioners for parabolic optimal controal problems. SIAM J Sci Comput 32, 1180-200.

17. Wu SL, Shi BC, Huang CM (2009) PararealRichardson algorithm for solving nonlinear ODEs and PDEs. Commun Comput Phys 6, 883-902.

18. Wu SL (2016) A second-order parareal algorithm for fractional PDEs. J Comput Phys 307, 280-90.

19. Wu SL (2015) Convergence analysis of some second- order parareal algorithms. IMA J Numer Anal 35, 1315-41.

20. Wu SL, Zhou T (2017) Fast parareal iterations for fractional diffusion equations. $J$ Comput Phys 329, 210-26.

21. Wu SL, Zhou $\mathrm{T}$ (2018) Parareal algorithms with local time-integrators for time fractional differential equations. J Comput Phys 358, 135-49.

22. Wu SL, Zhou $\mathrm{T}$ (2015) Convergence analysis for three parareal solvers. SIAM J Sci Comput 37, 970-92.

23. Wu SL (2016) Convergence analysis of the PararealEuler algorithm for systems of ODEs with complex eigenvalues. J Sci Comput 67, 644-68.

24. Wu SL (2018) Toward parallel coarse grid correction for the parareal algorithm. SIAM J Sci Comput 40, 1446-72.

25. Erbe LH, Freedman HI, Liu XZ, Wu JH (1991) Comparison principles for impulsive parabolic equations with applications to models of single species growth. J Austral Math Soc Ser B 32, 382-400.

26. Lakshmikantham V, Bainov DD, Simeonov PS (1989) Theory of Impulsive Differential Equations, World Scientific, Singapore. 